\title{
Innovative Designs for the Imaging Suite on Solar Orbiter
}

\author{
Frédéric Auchère ${ }^{a}$, Xueyen Song ${ }^{a}$, Frédéric Rouesnel $^{a}$, Thierry Appourchaux ${ }^{a}$, Jean-Jacques \\ Fourmon $^{a}$, Jean-Christophe Le Clec'h ${ }^{a}$, Michel Berthé ${ }^{a}$, \\ Jean-Marc Defise ${ }^{b}$,Emmanuel Mazy ${ }^{b}$, Pierre L. Rochus ${ }^{b}$ \\ Raymond Mercier $^{c}$, Marie-Françoise Ravet ${ }^{c}$ \\ ${ }^{a}$ Institut d'Astrophysique Spatiale, Bâtiment 121, Université Paris-Sud, 91405 Orsay, France; \\ ${ }^{b}$ Centre Spatial de Liège, Université de Liège, Av. du Pré-Aily, 4031 Angleur, Belgium; \\ ${ }^{c}$ Institut d'Optique Théorique et Appliquée, Bâtiment 503, Université Paris-Sud, 91403 Orsay, \\ France
}

\begin{abstract}
Orbiting around the Sun on an inclined orbit with a 0.2 UA perihelion, the Solar Orbiter probe will provide high resolution views of the Sun from various angles unattainable from Earth. Together with a set of high resolution imagers, the Full Sun Imager is part of the EUV Imaging suite of the Solar Orbiter mission. The mission's ambitious characteristics draw severe constraints on the design of these instruments. We present a photometrically efficient, compact, and lightweight design for the Full Sun Imager. With a 5 degrees field of view, this telescope will be able to see the global solar coronal structure from high viewing angles. Thermal solutions reducing the maximum power trapped in the High Resolution Imagers are also proposed.
\end{abstract}

Keywords: Solar Orbiter, EUV instruments

\section{INTRODUCTION}

The Full Sun Imager (FSI) is part of the Extreme Ultraviolet Imager suite on Solar Orbiter. Much like the Extreme-ultraviolet Imaging Telescope (EIT) on SOHO, FSI will both be a source of scientific discovery and a guide for the observations of the other instruments. With its 5 degrees field of view (FOV, see Figure 1), FSI will be the only instrument on Solar Orbiter capable of providing a global picture of the solar corona at any given time. The FOV of FSI is equal to 2 solar radii as seen from the perihelion (0.2 AU) so that the full Sun will be imaged even when the spacecraft will be pointed at the limb. The field of view of the High Resolution Imagers (HRI) is 17 arcminutes, which is equivalent to 3.4 arcminutes from Earth. Most solar features will not be visible in their entirety in such a small FOV, which will prevent from determining their geometry. Because of the inclination of the orbit of Solar Orbiter, FSI will have a direct and global view of the polar regions of the Sun, including polar plumes. The large FOV of FSI is necessary to identify the source regions of the plumes. A simulation of the solar minimum corona seen from $35^{\circ}$ of heliographic latitude is shown in the right panel of Figure 1. The multi-spectral capability that we propose to add will also permit to sample both the transition region and the average million degree corona, giving a more complete picture of the solar atmosphere. Even though Solar Orbiter is not dedicated to the observation of Coronal Mass Ejections (CME), it is likely that it will observe many such events. To make the connection between these events and the in-situ measurements it is required that FSI observes at a cadence of about 5 minutes, even if at a reduced resolution. During the first year of operations of EIT on SOHO, many CMEs were missed because too few images were taken, until the cadence of observation was increased in 1997. EIT images taken during offpoints of the SOHO spacecraft show very large transequatorial loops that extend outside the normal FOV. The FOV of FSI is up to $2 \mathrm{R}_{\odot}$, significantly more than the $1.4 \mathrm{R}_{\odot}$ of EIT, which will permit the study of these large coronal structures. The knowledge of the solar irradiance at high heliographic latitudes is of major importance for several applications in solar and heliospheric physics. A model of the heliospheric EUV flux developed by Auchere et al. ${ }^{1}$ showed that the EUV

Further author information: (Send correspondence to F.A.)

F.A.: E-mail: frederic.auchere@ias.u-psud.fr, Telephone: +33 (0)1 69858733

J.M.D.: E-mail: jmdefise@ulg.ac.be, Telephone:+32 43676668 

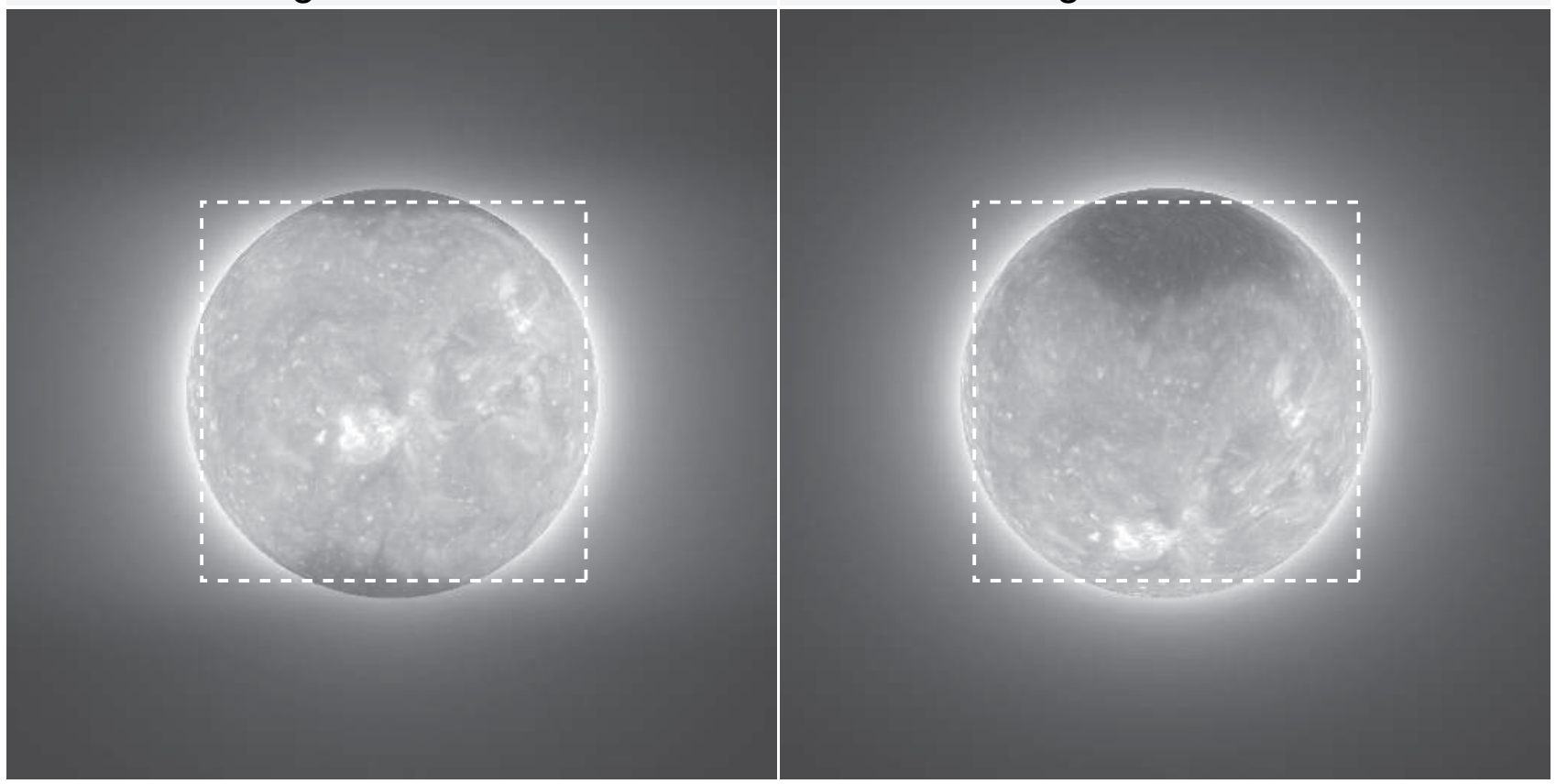

Figure 1. Simulations of the Sun as seen by FSI from two heliographic latitudes : $0^{\circ}$ (left) and $35^{\circ}$ (right). The size of the Sun corresponds to the perihelion of Solar Orbiter $(0.2 \mathrm{AU})$. The dashed squares represent the inner $2048 \times 2048$ pixels subfield that can be transmitted at the maximum resolution of $4.5^{\prime \prime} /$ pixel.

flux is strongly anisotropic, and is smaller by up to a factor of 2 above the solar poles compared to an equatorial viewpoint at the same distance. The observations of FSI will provide a direct test of this model. The Solar Orbiter strawman payload does not include an EUV spectral irradiance monitor. In a recent work, Dudok de Wit et al. ${ }^{2}$ showed that a limited set of spectral lines (typically 4 to 6 ) chosen by statistical analysis is sufficient to reconstruct the entire full Sun spectrum between $17 \mathrm{~nm}$ and $160 \mathrm{~nm}$. As we will see below, FSI could easily have the capability to observe in two passbands centered on $17.1 \mathrm{~nm}$ and $30.4 \mathrm{~nm}$. Although not the optimum choice of lines for spectrum reconstruction, a linear combination of these two will be a very good proxy of the solar EUV activity.

\section{INSTRUMENT CONCEPT}

The spectral intensity of the Sun is imposed by nature and does not depend on the distance of the oberver. Whether an instrument is located at $1 \mathrm{AU}$ or at $0.2 \mathrm{AU}$ does not change the exposure time need to achieve a given signal to noise ratio. The speed of a telescope is inversely proportional to its $f / D$ ratio. If two telescopes have identical detectors and identical apertures, the wide-field telescope will be faster than the high resolution telescope, in proportion to the square of the ratio between their angular resolutions. This is why FSI $\left(8.8^{\prime \prime} /\right.$ pixel) is instrinsically 310 times faster than HRI $\left(0.5^{\prime \prime} /\right.$ pixel $)$. Build with the technology used for EIT $\left(2.6^{\prime \prime} /\right.$ pixel $)$, HRI would be 7 times slower, while FSI would be 45 times faster. Going closer to the Sun does not improve the photometry of a high resolution telescope, but improves a lot that of a full Sun imager. Unlike the High Resolution Imagers, FSI will not lack photons. In addition to this purely geometric factor, the present design uses a single reflection, which significatively improves the total efficiency of the instrument. The wavelengths considered at present are $17.1 \mathrm{~nm}, 17.5 \mathrm{~nm}$ and $19.5 \mathrm{~nm}$. The final choice will be a tradeoff between temperature, sensitivity, photometry and technology. Based on these considerations, a photometric estimate at $19.5 \mathrm{~nm}$ shows that the pupil can be as small as 5 milimeters in diameter for 10 seconds exposures and a signal to noise ratio greater than 10 in most of the FOV. At the EUV wavelengths considered, this is still large enough not to be 
diffraction limited. The small pupill has the enormous advantage to solve the heat flux problem. At perihelion, only 0.67 Watt enter the intrument.

With a pupil 5 milimeters in diameter, two types of simple optical systems can provide spots of 9 arcseconds over a $5^{\circ}$ FOV. The first type is the Fresnel Zone Plate (FZP) cameras. The second family is that of single-mirror off-axis telescopes. One could also think of using a pinhole camera such as those used to record the early X-Ray images of the Sun, ${ }^{3}$ but the aperture would be too small for a reasonable exposure time, and diffraction would limit the resolution. FZP cameras have been studied and flown in the past to image the Sun in soft X-Rays, ${ }^{4},{ }^{5} .{ }^{6}$ More recently, Artzner et al. ${ }^{7}$ have proposed a variation of the FZP camera using a photon sieve ${ }^{8}$ instead of a classical FZP. The spot size at the focal plane of a FZP is equal to the width of the marginal zone. A $10 \mu \mathrm{m}$ spot size is therefore achievable with existing technology. However, if the spot itself is very narrow, more than $90 \%$ of the energy is spread in the extended wings of the PSF. The artefacts produced by the flat wings of the PSF are clearly visible in the images taken by Kramer et al. ${ }^{6}$ as bright disks centered on the main solar features. The multistep phase plates developed for keV X-Ray applications have an efficiency up to $55 \% .^{9}$ But an EUV version would be very challenging to fabricate, mainly because in the EUV no material can introduce $\pi / 2$ phase shift without absorbing significantly. We studied the properties of the PSF of calssical FZPs and photon sieves and found that in all cases, the extended wings were more pronounced than that of the corresponding single-mirror telescope. Also, it is not possible to baffle a FZP to protect it from the 25 solar constants heat flux.

Although very interesting because of its intrinsic small spot sizes and compactness we discarded the FZP camera because in comparison, the single-mirror off-axis telescope has a robust design providing optimum image quality in a reasonably compact package. The present design is illustrated by Figure 2. All the dimensions are linked to the choice of the detector, whose size determines the focal length of the mirror. It is not clear which detector will be chosen for Solar Orbiter. CCDs are said not to be suited to Solar Orbiter because of their sensitivity to radiations. At present, ESA baselines rad-hard APS-based cameras. We studied the present design assuming a $4096 \times 4096$ pixels detector with $10 \mu \mathrm{m}$ pitch. This is larger than the $2048 \times 2048$ detectors proposed by ESA in the strawman payload but as we will see, it adds extra capabilities and flexibility to the instrument.

A circular entrance pupil 5 millimeters in diameter is placed 700 millimeters in front of an off-axis ellipsoïdal mirror $(\mathrm{f}=450 \mathrm{~mm})$. The image is formed on a $4 \mathrm{k} \times 4 \mathrm{k}$ detector used in $2 \times 2$ binned mode. A thin-film aluminum filter $47 \mathrm{~mm}$ in diameter is placed $330 \mathrm{~mm}$ behind the pupil, where it receives a solar flux diluted by a factor 15 , which corresponds to only 1.66 solar constant at the maximum. The whole instrument fits in a $800 \times 150 \times 150 \mathrm{~mm}^{3}$ envelope, which represents a significant gain in volume compared to what is proposed by ESA in the strawman payload. The pupil is put $250 \mathrm{~mm}$ sunwards from the detector to account for the expected thickness of the sunshield and of the spacecraft front structure.

The reason for using a $4 \mathrm{k} \times 4 \mathrm{k}$ detector is twofold. First, the longer the focal length, the better the image quality. Second, as can be seen on Figure 3, the central part of the detector can be used in full-resolution mode with good images quality. This allows for imaging at a constant resolution all along the orbit of Solar Orbiter. When the spacecraft is at the perielion, the full detector is read and the images binned before being telemetered. When the spacecraft is farther from the Sun, only the central subfield (one quarter) of the detector is read and the images are kept unbinned. Note that only $2048 \times 2048$ pixels images are produced by this scheme, which ensures at constant data rate. Unlike what is currently planned in the mission profile, it is important that FSI can observe continuously, and not only around the perihelion.

Since the pupil is much smaller than the mirror, the beams imaged on the edge of the detector draw a slightly rounded square on the mirror. Making this latter square will save a factor $4 / \pi$ (or $27 \%$ ) in mass.

The current philosophy for the EUI suite is not to use any mechanism. However, with the addition of a filter wheel, and without altering the design, we can make FSI work in two wavelengths. It is a general property of mulilayers to have a second order peak in reflectivity a about half of the central wavelength. The Mo/Si multilayers developped for the $30.4 \mathrm{~nm}$ passbands of the UVI telescopes (see Fig. 5) have a narrow second order peak around $16.5 \mathrm{~nm}$, close to wavelength of the group of Fe IX/X lines. Great care was taken in designing this multilayer in order to keep this second order peak shortwards of the aluminum edge, so that it would not contaminate the main peak centerded on $30.4 \mathrm{~nm}$. However, if shifted by $0.5 \mathrm{~nm}$, the second oder peak would make for a highly reflective narrow bandpass to image the Fe IX/X lines. By fine-tuning the multilayer 


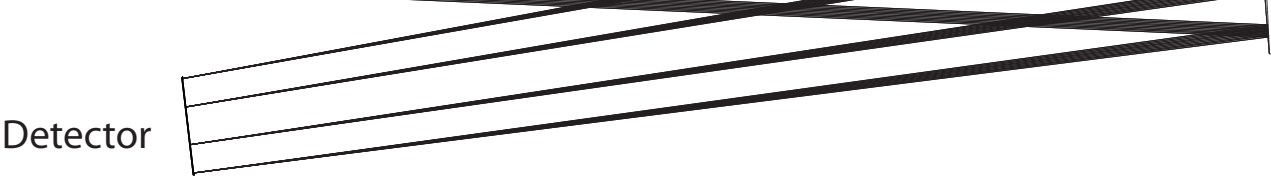

Figure 2. Raytracing of the FSI.

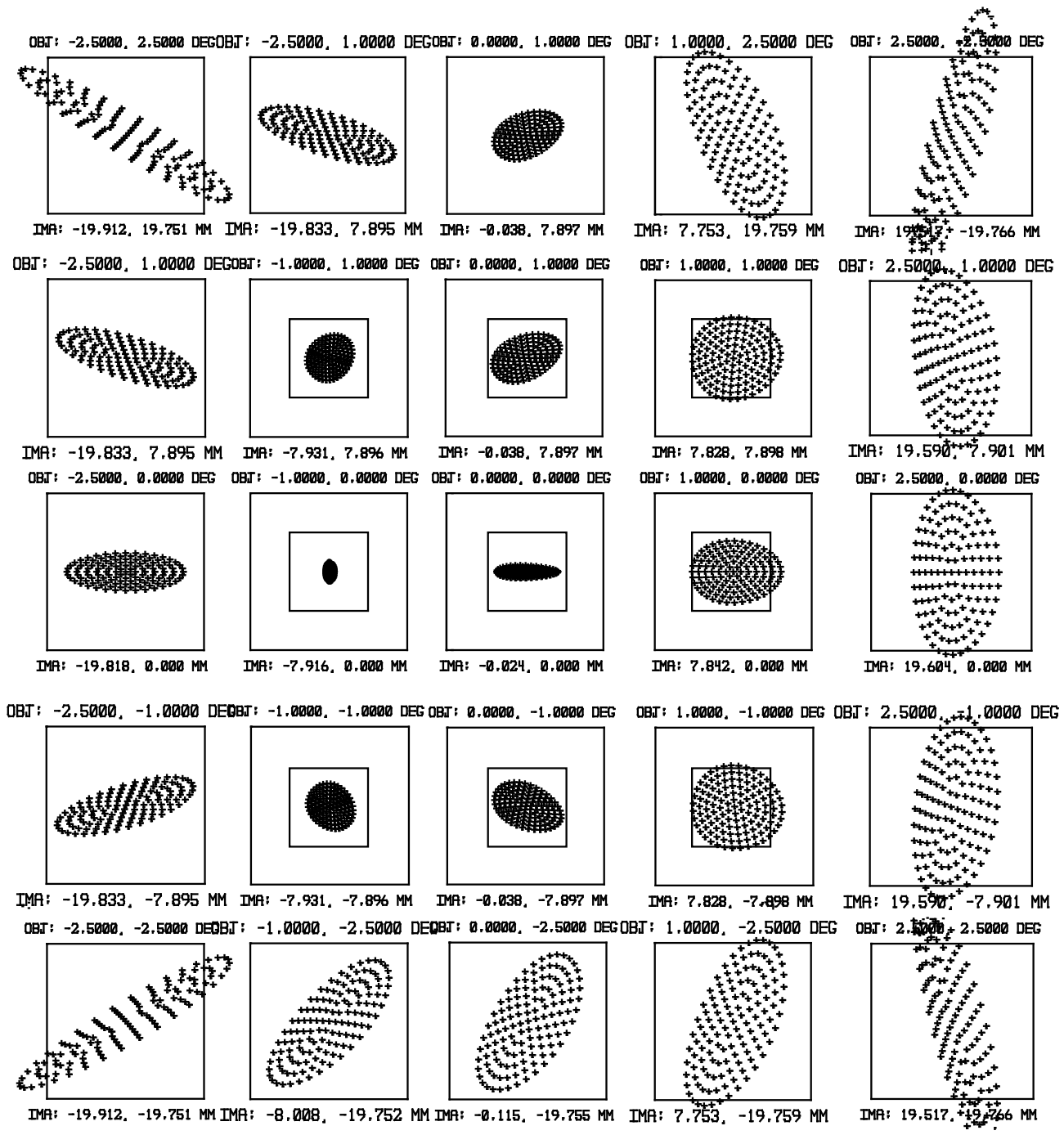

Figure 3. Spot diagrams across the FOV. The reference boxes are $20 \mu \mathrm{m}$ wide and represent the $2 \times 2$ binned pixels. The small boxes in the 9 inner fields represent non-binned pixels. The spots in the inner fields are within a non-binned pixel, which allows for high resolution imaging when Solar Orbiter will be far from the Sun (see also Figure 1). 


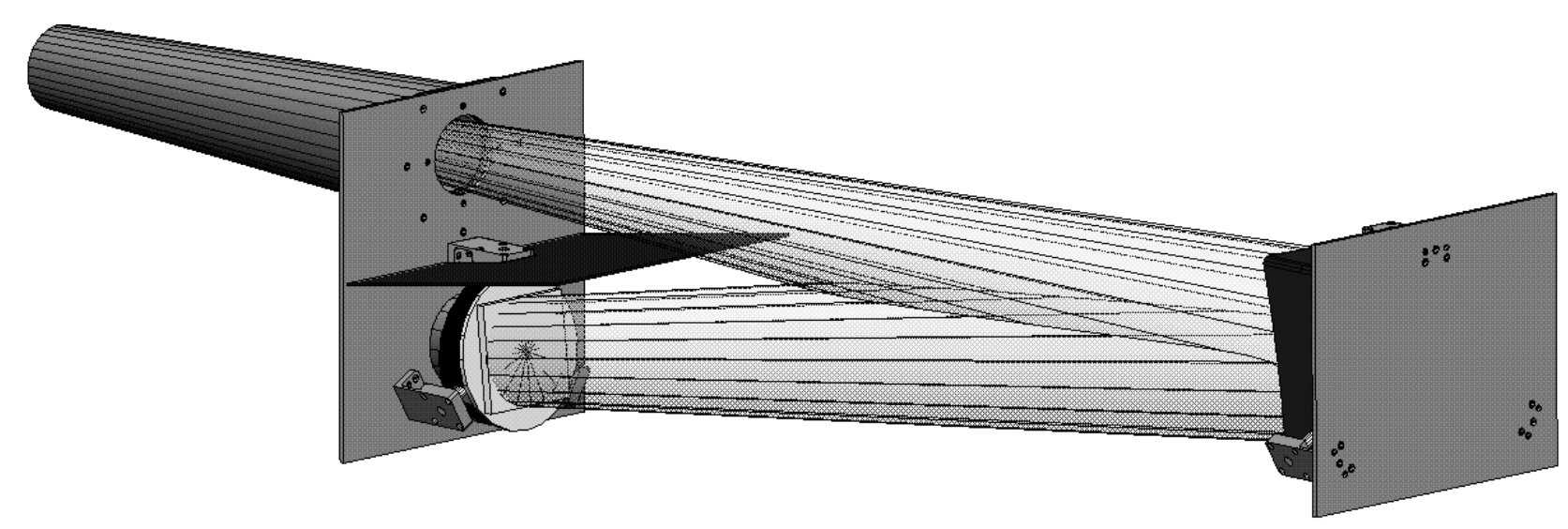

Figure 4. System concept of FSI. The box joining the two bulkheads is not shown. The front baffle is designed to be inserted in the thermal shield of the spacecraft.

properties, Ravet et al. ${ }^{10}$ managed to produce samples for which the two orders are centered on $17.1 \mathrm{~nm}$ and $30.4 \mathrm{~nm}$. Such a multilayer can be used to image the two wavelengths with a single aperture, the selection between the two orders being obained with two filters : one Zirconium filter to select the 17.1 band, and one Aluminium/Magnesium/Aluminum filter to select the $30.4 \mathrm{~nm}$ band. Equipped with a double-peaked coating and a filter wheel, FSI could image both the Fe IX/X lines (formation temperature around $1 \mathrm{MK}$ ) and the He II line $(80 \mathrm{kK})$. This way, FSI would provide the other instruments with a much more complete view of the EUV Sun, including all possible solar structures from the lower transition region to the corona. The $17.1 \mathrm{~nm}$ is ideal to sample the million-degree corona, while the $30.4 \mathrm{~nm}$ bandpass is best suited to detect network cells and prominences. It is important to note that the implementation of this multichannel capability is thermally very different than that used in EIT or TRACE. EIT and TRACE are equivalent to having four telescopes side by side. Using the same approach, a secod wavelength would mean an extra pupil, which would double the thermal load on the instrument. In the present design, we have a single pupil for the two wavelengths and there is no increase in thermal input.

An engineering study of FSI based on the concept presented in this paper is ongoing. Figure 4 shows a left-rear view of the present implementation. The box joining the two flanges is not shown.

\section{HEAT REJECTION SYSTEMS FOR HRI}

If the small aperture is the straightforward solution to limit the heat load in FSI, it can not be used in the case of the High Resolution Imagers (HRIs). Because of their much higher spatial resolution $\left(0.5^{\prime \prime} /\right.$ pixel), the HRIs need a much larger entrance pupil, at least 2 or 3 centimeters in diameter, which corresponds to 10.78 and 24.26 Watts at $0.2 \mathrm{AU}$ respectively. The need for photons goes against the necessity to limit the amount of power to be dissipated by the spacecraft. However, since the field of view of HRI is small $\left(17^{\prime}\right)$, a significant amount of the power entering the instrument is contained in the unobserved fields and can be rejected outside the instrument.

Specular baffles designed to reject unwanted radiations have been extensively studied. Baffles composed of stacked ellipsoids and hyperboloids are able to reject light entering the system under high incidence angles (30 degrees or more). Such baffles are suited to the observation of an object located close to a bright source. They have been recently considered in the framework of the Beppi-Colombo mission to reject the solar light susceptible to enter the telescopes observing Mercury. In our case, we need to reject the light coming from only 2.5 degrees around the viewing direction. In this case, a simple annular mirror positionned between the entrance pupil and the primary mirror can reject most of the incoming flux. Such heat rejection mirrors have been successfuly used in coronagraphs (for example in the LASCO $\mathrm{C} 2$ and $\mathrm{C} 3$ onboard $\mathrm{SOHO}^{11}$ ), and a heat rejection mirror is currently planned for the coronagraph present in the strawman payload of Solar Orbiter. The heat rejection mirrors used in coronagraphs are tipped parabolic mirros that image the solar disk in the plane of the external 


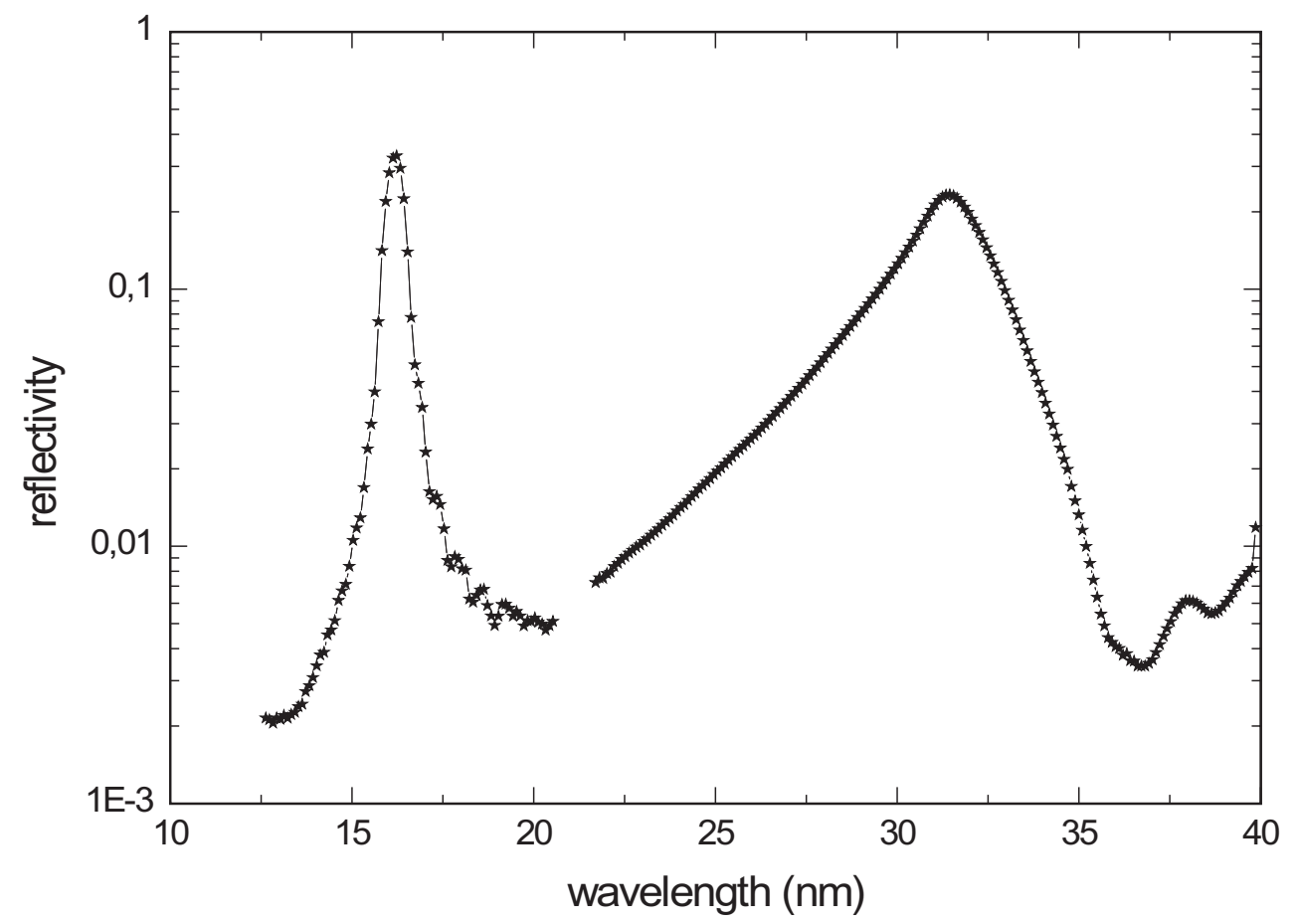

Figure 5. Reflectivity of a Mo/Si multilayer produced for the $30.4 \mathrm{~nm}$ passband of one of the UVI telescopes of the STEREO mission.

occulter. This configuration corresponds to the left diagram of Figure 6. In our case, the mirror doesn not need to be tipped since there is no occulter in the plane of the pupil. Since the spacecraft can be pointed at towards the limb of the Sun, the diameter $b$ of the image of the Sun needs to be half that of the pupil $(a)$. This implies that the distance $L$ between the pupil and the mirror, which is equal to the focal length of the mirror, is equal to $a / 4 \tan \alpha_{s}=a \times 11.45, \alpha_{s}$ being the solar radius seen from $0.2 \mathrm{AU}$. The mirror is therfore illuminated by 11 solar constants, whatever the diameter of the pupil. The outer diameter $d$ of the beam on the mirror is then $3 a / 2$, and the inner diameter is $e=a+2 L \tan \alpha_{c}$, where $\alpha_{c}$ is the half angle of the FOV. Assuming that the mirror is made of aluminum (90\% reflectivity), one finds that $45 \%$ of the incoming flux is rejected through the pupil, the rest being absorbed by the aluminum filter and by the structure of the baffle. If the aluminum filter were to be positionned in the opening of the mirror, then it would have to withstand 11 solar constants, which may not be realistic. This design has the further inconvenient of forming an image in the plane of the pupil. This is a source of damage to the instrimen and to the spacecraft itself in case this latter is offpointed further than 2.5 degrees from the center of the solar disk. Another configuration that overcomes these limitations is illustration by the middle diagram of Figure 6. If the pupil is positionned at the radius of curvature of a spherical mirror, then the pupil is imaged on itself whatever the distance betweeen the pupil and the mirror, and every ray entering the pupil goes out through the pupil. The mirror can be put at any distance from the pupil so that the flux density on the aluminum filter is diluted by an arbitrary factor. An image of the Sun is formed at the focal plane of the mirror in the middle of the baffle, but there is not more risk of damaging the instrument. A similar design uses a fundamental property of ellipses. The baffle consists of an elliptical mirror which foci are at the edge of the pupil (right diagnram of Figure 6). A ray entering the baffle tangent to the pupil will be reflected out tangent to the opposite edge of the pupil. This is the classical specular baffle design and corresponds to the first stage of the stacked baffle described by Kleinhans. ${ }^{12}$

\section{ACKNOWLEDGMENTS}

The author would like to thank J.-P. Delaboudinière for animated and stimulating fruitful discussions. 

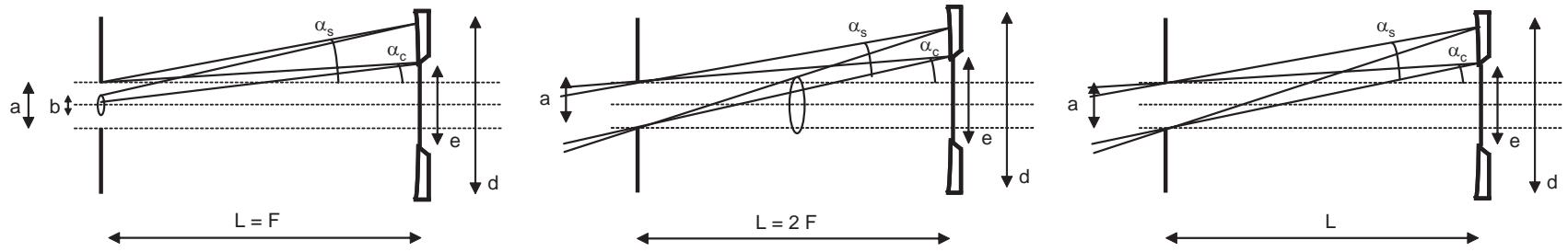

Figure 6. Three possible configurations for the heat rejection mirror of HRI.

\section{REFERENCES}

1. F. Auchère, J. W. Cook, J. S. Newmark, D. R. McMullin, R. von Steiger, and M. Witte, "The Heliospheric He II 30.4 nm Solar Flux During Cycle 23," Astrophys. Journ. 625, pp. 1036-1044, June 2005.

2. T. Dudok de Wit, J. Lilenstein, J. Aboudarham, A. P.-O., and M. Kretzschmar, "Retrieving the solar EUV spectrum from a reduced set of spectral lines," Annales Geophysicae, 2005.

3. R. L. Blake, T. A. Chubb, H. Friedman, and A. E. Unzicker, "Interpretation of X-Ray Photograph of the Sun.," Astrophys. Journ. 137, pp. 3-+, Jan. 1963.

4. G. Elwert, "X-Ray Picture of the Sun Taken with Fresnel Zone Plates," in IAU Symp. 35: Structure and Development of Solar Active Regions, pp. 439-+, 1968.

5. J. H. Dijkstra, W. de Graaff, and L. J. Lantwaard, "Construction of Apodised Zone Plates for Solar X-Ray Image Formation," in IAU Symp. 41: New techniques in Space Astronomy, pp. 207-+, 1971.

6. G. Kraemer, G. Elwert, H. J. Einighammer, H. Braeuninger, and H. H. Fink, "Imaging of solar active regions with Fresnel zone plates," in X-ray imaging; Proceedings of the Seminar, Reston, Va., April 18-21, 1977. (A78-40248 17-35) Bellingham, Wash., Society of Photo-Optical Instrumentation Engineers, 1977, $p$. 79-84., pp. 79-84, 1977.

7. G. E. Artzner, J. P. Delaboudiniere, and X. Song, "Photon sieves as EUV telescopes for Solar Orbiter," in Innovative Telescopes and Instrumentation for Solar Astrophysics. Edited by Stephen L. Keil, Sergey V. Avakyan. Proceedings of the SPIE, Volume 4853, pp. 158-161 (2003)., pp. 158-161, Feb. 2003.

8. L. Kipp, M. Skibowski, R. L. Johnson, R. Berndt, R. Adelung, S. Harm, and R. Seemann, "Sharper images by focusing soft X-rays with photon sieves," Nature 414, pp. 184-188, Nov. 2001.

9. E. di Fabrizio, F. Romanato, M. Gentili, S. Cabrini, B. Kaulich, J. Susini, and R. Barrett, "High-efficiency multilevel zone plates for keV X-rays," Nature 401, pp. 895-898, Oct. 1999.

10. M.-F. Ravet Private communication, 2005.

11. G. E. Brueckner, R. A. Howard, M. J. Koomen, C. M. Korendyke, D. J. Michels, J. D. Moses, D. G. Socker, K. P. Dere, P. L. Lamy, A. Llebaria, M. V. Bout, R. Schwenn, G. M. Simnett, D. K. Bedford, and C. J. Eyles, "The Large Angle Spectroscopic Coronagraph (LASCO)," Sol. Phys. 162, pp. 357-402, 1995.

12. W. A. Kleinhans, "Skew ray results for condensing light pipes and reflective baffles," Appl. Optics 15, pp. 2437-2439, Oct. 1976. 